\title{
Impact of Child Interaction With Food Preparation on Vegetable Preferences: A Farm-Based Education Approach
}

\author{
Isabelle L. Crary, $\mathrm{BA}^{1, \dagger}$; Nicole M. Ardoin, $\mathrm{PhD}^{2}$; Christopher Gardner, $\mathrm{PhD}^{3}$
}

\begin{abstract}
Objective: To identify the impact of child involvement in vegetable preparation on vegetable preference and attitudes toward eating vegetables.

Design: Pre-post mixed-methods.

Setting: Food and Farming Camp at a nonprofit urban farm in Sunnyvale, California.

Participants: Camp participants aged $8-10$ years $(n=34$ girls, $n=12$ boys $)$, and aged $11-14$ years $(n=19$ girls, $\mathrm{n}=4$ boys).

Interventions: Involvement in vegetable preparation through harvesting, cutting, cooking, and seasoning before consumption. Interviews identified camper perception of vegetable preference and involvement in preparation.

Main Outcome Measures: Change in vegetable preference from baseline with and without involvement in vegetable preparation. Attitudes toward involvement in vegetable preparation.

Analysis: Adjustment of preferences to baseline followed by tests of hypotheses to identify differences with involvement. Thematic, qualitative coding to identify prevalent themes within interview responses.

Results: Younger campers preferred vegetables they prepared $(P<0.05)$, except for carrots. Campers were more likely to choose vegetables they prepared $(P<0.05)$. Campers of both age groups were curious to try their vegetable creations and described feelings of pride and responsibility related to preparing vegetables.

Conclusions and Implications: Involvement with food preparation, in particular in a garden-based setting, may provide an accessible method to improve child vegetable preference.

Key Words: cooking, vegetable intake, gardening, school-aged children (J Nutr Educ Behav. 2022;54:46 -55 .)
\end{abstract}

Accepted August 15, 2021. Published online November 11, 2021.

\section{INTRODUCTION}

The state of childhood health and nutrition in the US is far from ideal. Obesity, type 2 diabetes, and hypertension rates are higher than ever among adolescents. ${ }^{1-4}$ Diets high in fats, sugar and sodium are associated with increased risk of obesity and cardiovascular disease in adolescents. $^{5-6}$ On the other hand, increased vegetable intake is correlated with decreased mortality, risk of cardiovascular disease, cancer, and weight gain in adults. ${ }^{7-14}$ Given that dietary patterns developed in childhood often carry into adulthood, developing a diet high in fruits and

\footnotetext{
${ }^{1}$ University of Washington School of Medicine, University of Washington, Seattle, WA

${ }^{2}$ Graduate School of Education, and Woods Institute for the Environment, Stanford University, Stanford, CA

${ }^{3}$ Stanford Prevention Research Center, Department of Medicine, Stanford University, Stanford, CA

$\dagger$ Isabelle L. Crary was affiliated with the Stanford Prevention Research Center, Department of Medicine, Stanford University at the time this study was completed.

Conflict of Interest Disclosure: The authors have not stated any conflicts of interest.

Address for correspondence: Isabelle L. Crary, BA, University of Washington School of Medicine, 1959 NE Pacific St, Seattle, WA 98195; E-mail: icrary@uw.edu

(C) 2021 The Authors. Published by Elsevier Inc. on behalf of Society for Nutrition Education and Behavior. This is an open access article under the CC BY-NC-ND license (http:// creativecommons.org/licenses/by-nc-nd/4.0/)

https://doi.org/10.1016/j.jneb.2021.08.009
}

vegetables in adolescence can improve intake in adulthood. ${ }^{15}$

Despite these benefits, children in the US consume far fewer servings of vegetables and fruits than recommended. The 2020-2025 Dietary Guidelines for Americans recommends children eat between 1 and 4 cups Eq of vegetables a day, depending on age and gender. ${ }^{16}$ However, the current overall average for children aged 2 -19 years is only 0.90 cup Eq a day. Of these, 0.30 cup Eq are potatoes, 0.20 cup Eq are tomatoes (including processed tomato products such as pizza sauce), 0.26 cup $\mathrm{Eq}$ are of red and orange vegetables, and only 0.06 cup Eq are of dark greens. ${ }^{17}$ In a similar analysis of childhood nutrition, researchers discovered that $28 \%$ of the vegetables consumed by children were french fries. ${ }^{18}$ Taking into account the health benefits of vegetable consumption along with the notably low vegetable consumption among adolescents in 
the US, interventions focused on increasing vegetable consumption may play key roles in addressing childhood health and nutrition concerns in the US.

Cooking and gardening interventions have become popular methods by which to address low vegetable consumption globally. ${ }^{19}$ Children's participation in garden-based programs that include activities such as taste-testing vegetables, harvesting, and using garden produce to make snacks can improve both vegetable consumption and preference. ${ }^{20,21}$ Furthermore, several studies have shown that supplementing nutrition education programs with gardenbased education is more successful at improving vegetable consumption and/or preference than a nutrition education program alone. ${ }^{22-25}$

Several studies have focused on children's involvement in other forms of food preparation and interaction, such as cooking, on child vegetable preferences. ${ }^{26,27}$ Cooking interventions commonly involve participants in food preparation activities, including but not limited to cutting, baking, cooking, seasoning, and tasting, and then evaluating their preferences and/or consumption of self-prepared food. Cooking classes have positively affected child vegetable preferences. ${ }^{28}$ Two studies showed that integrating cooking activities into garden-based interventions increased vegetable consumption among adolescents in the US. $^{20,29}$ However, the success of cooking and gardening interventions is not universal, suggesting a need for further exploration of the impact of cooking interventions on vegetable consumption outcomes. ${ }^{30}$

To our knowledge, studies to date have not compared the cumulative effects of vegetable preparation processes on vegetable preference or consumption, especially among adolescent populations. Consequently, this research is designed to investigate 2 questions: (1) How does child interaction with the vegetable preparation process-through harvesting, cutting, cooking, and seasoninginfluence their vegetable taste preferences? (2) Why do children prefer certain vegetables to others within the camp setting?

\section{METHODS}

This exploratory mixed-methods research took place at Full Circle Farm's Food and Farm Camp during the summer of 2017. Full Circle Farm is an 11-acre community-based sustainable farm located in Sunnyvale, California. The camp was specifically held at a small community garden located at the farm. The farm partners with Stanford University to research a range of topics related to children's health and wellness, including child vegetable preferences, environmental education, and nutrition. The initial protocol for this human subjects research was approved by the Stanford University Institutional Review Board in 2012, with annual renewals. The year-long renewal for this study was granted in May, 2017. Children attended camp for 1-week intervals, during which they completed precamp and postcamp vegetable preference surveys, along with daily vegetable preference surveys to evaluate how vegetable preferences changed in relation to involvement in vegetable preparation and consumption.

Participants included youth from 3 sessions of camp, each session of which was 1 week: 2 sessions of 8 - to 10-year-old campers and 1 session of 11- to 14-year-old campers. These groups of campers will be referred to as the younger and older campers, respectively. Any camper who signed up for camp was eligible to participate in the research. Parents gave written consent, and children gave written assent for participation in research studies before attending camp. Participants represented a nonrandomized convenience sample that was not predetermined but rather limited to the registration capacity of the camp. Campers who chose not to participate in camp activities or did not complete all data collection were excluded from the sample $(n=4)$. Qualitative data were used to complement quantitative data and enhance the descriptive validity of findings. ${ }^{31}$

\section{Quantitative Data Collection}

Three sources of interconnected quantitative data were analyzed for this research.
Precamp and postcamp surveys were used to collect vegetable preference data on the first day (Monday) and last day (Friday) of each session of camp. The survey asked campers to rate 6 vegetables (zucchini, kale, carrots, tomatoes, green beans, and bell peppers) on a 5-point Likert-type scale $\left(1=\right.$ yuck!; $5=$ yum!). ${ }^{20,21}$ The bell pepper served as the control vegetable as campers ranked the vegetable in the precamp and postcamp surveys but were not exposed to that vegetable over the camp session. The control vegetable was used to enhance internal validity and support conclusions of the potential influence of farm camp activities on preferences.

Campers also completed food scientist journals during snack and lunch periods in which they had the opportunity to consume any of the 5 measured vegetables. The journals prompted campers to indicate how they were involved in preparing the vegetable (eg, harvest, cut, cook, season, or only ate it) and to rate the vegetable on the same 5-point Likerttype scale as on the precamp and postcamp preference survey.

Lastly, researchers recorded which vegetables campers chose during lunch. After campers prepared lunch, researchers placed all prepared items on a common table for consumption. Campers could choose any of the foods available for lunch, which included foods they had helped to prepare as well as those they did not. Observational data were collected for the foods campers helped to prepare, and the foods campers chose at lunch.

\section{Qualitative Data Collection}

Semistructured interviews were conducted with individual campers over the 3 sessions of camp. All interviews were recorded on a digital recording device after campers gave verbal consent for recording before each interview. For each session of camp, 8-12 interviews were completed. All interviews were conducted at the end of the camp session (Thursday or Friday) to ensure that campers would be able to discuss camp with sufficient knowledge and experience and that the researcher had ample time to 
develop rapport with the campers. ${ }^{32}$ Interviewees included an equal number of campers with vegetable preferences in the top and bottom halves of the camp group (deemed veggie lovers and veggie skeptics). Campers chosen for interviews represented the camp demographics for age and gender. None of the campers who were asked to participate in interviews declined to do so. Camper interviews were conducted to the point when each successive interview no longer revealed novel themes but, rather, augmented and supported reflections described in prior camper interviews. ${ }^{33}$

Each interview followed a basic, semistructured protocol. ${ }^{34}$ Interview questions included open-ended questions regarding camp experience, informational questions about vegetable-related eating experience, and hypothetical questions addressing food likes and dislikes. The interview protocol was piloted through 3 interviews with younger campers to ensure item clarity, define appropriate geolocations on the farm, and confirm interview timing. ${ }^{34}$

Quantitative data analysis. This exploratory investigation included quantitative data collection. All handwritten numerical data were entered into 2 separate spreadsheets and compared with ensure consistency and accuracy of the manual data entry. Data were then uploaded to Stata for the remainder of the statistical analyses (version 16, StataCorp LLC, 2019). Campers served as their own control. Presurvey results were used as a baseline to evaluate changes in vegetable preference. ${ }^{35-37}$ The Shapiro-Wilks test was used to test for normality in the data. As data for vegetable preferences were not normally distributed consistently, nonparametric tests were chosen to analyze these data. The Wilcoxon signed-rank and Mann-Whitney U tests were used for precamp and postcamp survey preferences and preferences related to vegetable involvement, respectively. The Wilcoxon signed-rank test was also used to evaluate the precamp and postcamp preferences for the control vegetable to ensure that preferences for a vegetable to which children were not exposed did not change significantly throughout the camp session. A significant change in the control vegetable would bring into question the impact of in-camp exposure and consumption on vegetable preference. For binomial data, 1-sample proportions tests were used to obtain exact $P$ values. The threshold for significance was set to $P=0.05$. Adjustments were not made for multiple testing because of the study's exploratory nature.

Qualitative data analysis. After interviews were transcribed, personally identifiable data were removed. All interviews were reviewed to seek themes related to child attitudes and preferences in involvement in food preparation and consumption. This review generated a list of 65 inductive, value, descriptive, in vivo codes and external codes. Two passes of coding were carried out by the primary researcher using NVivo software (version 11, QSR International, Inc, 2016). In the first pass of coding, primarily inductive, value codes were applied. In the second pass of coding, the majority of descriptive codeswhich, for example, identified how children described their food and how they were involved in preparation-were applied. ${ }^{38}$ The coding chart developed by the primary researcher included classification of code type, frequency of code, and an example quote of each code. The coding chart was not considered complete until no new codes were added as subsequent interviews were coded. ${ }^{39}$ Analytic memos addressing successes, areas for improvement, and evolving propositions regarding themes in the data were written after each coding pass. ${ }^{40,41}$

Matrix generation using NVivo software followed 2 complete rounds of coding as a means of initiating pattern recognition and theme development from coded transcripts. ${ }^{40}$ Building from propositions developed in analytic memos, content-analytic matrices were developed to compare codes pertaining to either internal (personal) or external (environmental) reasons campers expressed a preference for certain vegetables. For example, some codes described internal determinants included novelty, hard work, and earn, whereas codes for external preferences included fresh, organic, and negative (-) control. Verbatim quotes filled the boxes of each matrix. ${ }^{40}$ The results of the qualitative analysis included a collection of related themes addressing how children view vegetables they help prepare.

\section{RESULTS}

Demographics are described in Table 1. There were 3 separate camp sessions, each of which was 1 week in length. Two camp sessions were designed for primarily younger campers, whereas 1 session of camp was designed for older campers, a difference that is reflected in the number of campers per age group. Both age groups included a disproportionate number of girls, with females constituting $77 \%$ of the overall population registered for camp. Individual interviews were conducted with 29 individuals, representing $42 \%$ of the total camp population. Interviews ranged from 4 to 10 minutes, with a mean of 6.5 minutes.

\section{Vegetable Preference}

Table 2 highlights the changes in vegetable preference from the beginning to the end of the camp session. As zucchini, kale, and tomatoes had the lowest baseline preference among campers, these vegetables provided the greatest potential for improved preference. Significant improvements occurred in preference from the beginning compared with the end of the week for both age groups for zucchini $(P=0.002$ and $P<$ $0.001)$, kale $(P=0.002$ and $P=0.04)$, and tomatoes $(P=0.03$ and $P=0.02)$, with $P$ values presented for the younger age group and older age group, respectively. For the 2 initially mostpreferred vegetables, carrots, and green beans, there was no significant improvement in vegetable preference over the camp session (carrots: $P=0.31$ and $P=0.55$, green beans: $P=0.40$ and $P=0.72$; for younger and older campers, respectively). During the camp session, campers were not exposed to the control vegetable, bell pepper, and no significant difference in preference was found at the end of the camp session $(P=0.90$ and $P=0.10$, for the younger and older 
Table 1. Age and Gender Demographics for Campers Participating in Gardening/Cooking Intervention

\begin{tabular}{llccc}
\hline Age Group $^{\text {a }}$ & \multicolumn{1}{c}{ Variables } & Girls & Boys & Total \\
Younger & Total campers & 34 & 12 & 46 \\
& Number interviewed (\%) & $11(32 \%)$ & $6(50 \%)$ & $17(37 \%)$ \\
Older & Total campers & 19 & 4 & 23 \\
& Number interviewed (\%) & $10(53 \%)$ & $2(50 \%)$ & $12(52 \%)$ \\
Total & Total campers & 53 & 16 & 69 \\
& Number interviewed (\%) & $21(40 \%)$ & $8(50 \%)$ & $29(42 \%)$ \\
\hline
\end{tabular}

aYounger includes campers aged 8-10 years; older includes campers aged 11-14 years.

Table 2. Preintervention and Postintervention Vegetable Preference Data for Campers Participating in Gardening/ Cooking Intervention

\begin{tabular}{|c|c|c|c|c|}
\hline Vegetable & Age Group (n) ${ }^{a}$ & Presurvey, Mean (SD) ${ }^{\mathrm{b}}$ & Postsurvey, Mean (SD) ${ }^{\mathrm{b}}$ & $\boldsymbol{P}$ \\
\hline \multirow[t]{2}{*}{ Zucchini } & Younger (43) & $3.1(1.5)$ & $3.8(1.4)$ & $0.002^{\circ}$ \\
\hline & Older (21) & $3.3(0.9)$ & $4.5(0.8)$ & $<0.001^{\circ}$ \\
\hline \multirow[t]{2}{*}{ Carrot } & Younger (45) & $4.3(1.1)$ & $4.4(1.0)$ & 0.31 \\
\hline & Older (22) & $4.2(0.9)$ & $4.4(0.9)$ & 0.55 \\
\hline \multirow[t]{2}{*}{ Kale } & Younger (42) & $3.1(1.2)$ & $3.8(1.3)$ & $0.002^{\circ}$ \\
\hline & Older (21) & $3.3(1.3)$ & $4.1(1.0)$ & $0.04^{c}$ \\
\hline \multirow[t]{2}{*}{ Tomato } & Younger (45) & $3.7(1.6)$ & $3.9(1.4)$ & $0.03^{c}$ \\
\hline & Older (22) & $3.7(1.4)$ & $4.3(1.0)$ & $0.02^{c}$ \\
\hline \multirow[t]{2}{*}{ Green bean } & Younger (43) & $3.9(1.3)$ & $3.9(1.1)$ & 0.40 \\
\hline & Older (22) & $4.0(1.1)$ & $4.0(1.1)$ & 0.72 \\
\hline \multirow[t]{2}{*}{ Bell pepper } & Younger (38) & $3.4(1.5)$ & $3.4(1.6)$ & 0.90 \\
\hline & Older (22) & $3.5(1.5)$ & $3.8(1.3)$ & 0.10 \\
\hline
\end{tabular}

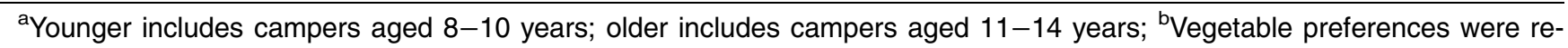
corded using a 5-point Likert-type scale (1, yuck!; 5 , yum!); ${ }^{\mathrm{c}} P<0.05$, as calculated using the Wilcoxon signed-rank test.

age groups, respectively). Overall, preference improved most for the vegetables with the lowest starting preferences and consequently the most room for improvement.

\section{Involvement and Vegetable}

\section{Preferences}

Table 3 presents the change of vegetable preference from baseline with and without involvement in vegetable preparation. Among the younger campers, involvement in vegetable preference was correlated with a significant improvement in vegetable preference compared with baseline for most vegetables. Vegetable preference improved when the younger campers were involved in preparing zucchini $(P<0.001)$, kale $(P=0.013)$, tomatoes $(P=0.004)$, and green beans $(P=0.010)$. The magnitude of the shift varied between vegetables, with zucchini showing the greatest increase in preference with involvement in preparation compared with baseline. As illustrated above in Table 2 , the baseline preference among younger

campers for zucchini was the lowest of all vegetables tested. Vegetable preference did not show improvement with involvement for carrots $(P=0.75)$. As noted earlier, the baseline preference for carrots was highest out of all vegetables, indicating little room for preference improvement.

\section{Involving children in vegetable preparation may improve vegetable preference.}

Among the older campers, no significant difference in vegetable preference was evident when campers were involved in food preparation. The qualitative data address potential age-based differences. Children's past experiences cooking and preparing food at home varied between age groups. Campers in the younger age group expressed that the opportunity to be involved in preparing food was a unique and cherished opportunity; this expression was more positive than for the older age group, which had experience assisting with food preparation. Younger campers generally described their parents' responsibility for at-home food preparation. One younger female, expressing this perspective, noted.

I don't really get to cook or do [any] of that stuff. I'm mostly just doing homework and getting the vegetables from my mom and here [at camp] I'm actually involved in the cooking.

This is not to say that the younger campers were not involved in preparation in any way at home. However, their involvement was often limited to chore-like activities, described by interviewees by comments such as: "I usually just wash" or "set[ting] the table."

The younger campers' parents were trusted with, the more dangerous or complex activities, such as cutting and cooking at the stovetop. One female camper described this difference in her own life, noting that, "It's usually my mom and 
Table 3. Effect of Involvement in Vegetable Preparation on Change in Vegetable Preference From Baseline

\begin{tabular}{|c|c|c|c|c|}
\hline Vegetable & Age $(n)^{a}$ & Involvement (No. of Exposures) ${ }^{b}$ & Mean Change (SD) & $\boldsymbol{P}$ \\
\hline \multirow[t]{4}{*}{ Zucchini } & Younger (46) & No involvement (96) & $0.31(1.6)$ & $<0.001^{\mathrm{C}}$ \\
\hline & & Involvement (39) & $1.3(1.3)$ & \\
\hline & Older (23) & No involvement (57) & $0.7(1.0)$ & 0.47 \\
\hline & & Involvement (25) & $0.9(1.0)$ & \\
\hline \multirow[t]{4}{*}{ Carrot } & Younger (45) & No involvement (98) & $-0.1(1.1)$ & 0.75 \\
\hline & & Involvement (38) & $0.1(1.4)$ & \\
\hline & Older (23) & No involvement (65) & $-0.2(1.0)$ & 0.99 \\
\hline & & Involvement (17) & $-0.1(1.2)$ & \\
\hline \multirow[t]{4}{*}{ Kale } & Younger (46) & No involvement (93) & $0.2(1.6)$ & $0.013^{c}$ \\
\hline & & Involvement (57) & $0.8(1.6)$ & \\
\hline & Older (23) & No involvement (65) & $0.2(1.5)$ & 0.10 \\
\hline & & Involvement (19) & $0.8(1.4)$ & \\
\hline \multirow[t]{4}{*}{ Tomato } & Younger (46) & No involvement (81) & $-0.1(1.0)$ & $0.004^{c}$ \\
\hline & & Involvement (48) & $0.4(1.2)$ & \\
\hline & Older (23) & No involvement (52) & $0.3(1.1)$ & 0.60 \\
\hline & & Involvement (35) & $0.4(0.9)$ & \\
\hline \multirow[t]{4}{*}{ Green bean } & Younger (41) & No involvement (83) & $-0.6(1.4)$ & $0.010^{c}$ \\
\hline & & Involvement (43) & $0.1(1.5)$ & \\
\hline & Older (23) & No involvement (54) & $-0.3(1.1)$ & 0.42 \\
\hline & & Involvement (20) & $-0.1(1.3)$ & \\
\hline
\end{tabular}

${ }^{\text {a}}$ Younger includes campers aged 8-10 years; older includes campers aged 11-14 years; ${ }^{\mathrm{b}}$ No involvement indicates the camper ate the vegetable but did not participate in the preparation of this vegetable. Involvement means the camper either harvested, cooked, chopped and/or seasoned the vegetable; ${ }^{\mathrm{c}} P<0.05$, as calculated using the Mann-Whitney $U$ test.

dad on the stove and with the big knives ... Sometimes I cut, but I usually just wash. At home your parents make it for you." Furthermore, the younger campers contrasted their experiences in preparing the camp vegetables vs those at home, mentioning their enhanced involvement at camp:

I help more at the farm than I help at home. I usually just help chop at home, but here I help harvest. I help chop. Sometimes I even help ... actually cook the food.

The idea of involvement in preparation being a unique, novel experience pervaded the younger camper dialogues.

In contrast, the older campers did not draw as sharp a contrast between their experiences at camp and home. The older campers were more familiar with the types of involvement and, concurrently, had more responsibilities at home. Older campers mentioned responsibilities at home and camp as being similar: "I sometimes take [the food] out if my parents want me to set the table, chop some stuff up, wash it, and follow it to the table." This camper described the process of helping with the full preparatory process at home, a task that many of the younger campers saw as new.

The older campers had home-based responsibilities and opportunities far more complex than those of the younger campers. One older male camper mentioned how he and his siblings make pizzas for the entire family: "Sometimes my sisters and I make pizzas together, so I chop some vegetables." Another explained how he had "done a 3-course meal once" and went on to explain the ingredients, seasonings, and spices in each of the courses. As evidenced through the older camper interviews, the level of involvement in the preparation, coupled with the complexity of the food they cooked at home, appeared notably greater than that of the younger campers. This lack of novelty may address why being involved in preparing vegetables did not significantly change the preferences of the older camp group, yet it did with the younger campers.

\section{Involvement and Vegetable Choice}

Campers were more likely to choose vegetables they helped prepare, either by harvesting, cutting, cooking, or seasoning, compared with foods prepared by other campers. Of the vegetables available at camp, the younger campers chose an average of $55.8 \%$ (SD, 28.1) of vegetables they helped prepare themselves and only $35.4 \%$ (SD, 17.9) of vegetables prepared by other campers $(P<0.001)$. The older-group campers chose $74.4 \%$ (SD, 19.1) of self-prepared vegetables and $52.3 \%$ (SD, 15.4) of the vegetables prepared by others $(P<$ 0.001). Furthermore, for 1 snack period during each camp session, campers had the opportunity to make their own tomato-based salsa. In addition, 33 of $44(75 \%, P<0.001)$ of the older campers and 21 out of 22 (95\%, $P<0.001)$ of the younger campers chose to eat the salsa they prepared.

Camper Perspective Regarding Vegetable Choice and Preference

Qualitative data gives further insight into why campers may have expressed a preference for certain vegetables within the camp setting. The theme of agency, which had many facets, including independence, curiosity, control, and pride, was 
pervasive across children's discussions of the food they eat at camp. The following analysis considers the voices of the campers themselves when providing further context to the previously presented quantitative results. These qualitative data reinforce the 2 primary findings previously discussed that (1) increases in the likelihood to choose one's vegetables in both age groups to co-occur with food preparation, and (2) the increase in vegetable preference, overall, among members of the younger age group.

Unlike findings from the quantitative analyses regarding the change in camper preferences across age groups, with some exceptions, camper comments and viewpoints related to the food they helped prepare were similar in the 2 age groups. The similarities in observations will be discussed first, followed by a brief discussion of differences between age groups.

Control. Campers enjoyed the opportunity to have physical control over the food they ate at camp. As discussed, the older campers often had more responsibilities and familiarity with being involved in preparing food at home compared with the younger age group. Nonetheless, both age groups expressed how the ability to control every step of the preparation process, from picking the vegetable to cooking it on the outdoor stove, was a novel and enjoyable experience. One older camper commented:

I think I have more control over the food at camp. Even though there are a lot of different things people are making, the thing that I make myself is ... completely mine. At home, we don't grow the food either, so ... control is taken away.

Although the camper noted that she did indeed have experience preparing food, she explained that she was able to exert a greater level of control at camp.

Among both age groups, campers noted that they appreciated having control over the food they ate. Whether it was through harvesting vegetables from the ground or adding new flavors to their food, they noted that the extent of control they were able to assert was above and beyond what they would have been able to do at home. In addition to having greater involvement throughout the process of preparing the food, campers also noted how they had more control over adding flavoring, such as spices, at various stages, personalizing the vegetables to their liking. One camper commented:

You can decide what you want to add to it to make it more appetizing for you. Like, you can add different spices if you want it spicy or if you want it sweet you could add cinnamon or something.

Certainty and uncertainty. Campers explained how they often preferred foods they helped prepare as they could then be more certain of how the foods would taste. By contrast, they were uncertain whether they would like the vegetables that others prepared. When describing the food he prepared at camp, an older boy noted,

Normally if I make a food, it generally tastes better to me because I know exactly what's in it and exactly how we prepared it. Also I feel good because I made it.

Similarly, in reference to the food she helped prepare, a younger female camper commented, "It tastes how I like it." These campers suggested that involvement in food preparation might provide a mechanism for enhancing preferences, as campers crafted the food to their liking. They may have created a dish they already knew they would enjoy, for example, although no significant, quantitative improvement in preference was evident with the older campers, the qualitative observations indicated value in the opportunity for greater certainty in how their food would taste.

Campers expressed the opposite sentiment with regard to vegetables with which they were not involved in preparing. When describing her choice to eat the salsa she helped prepare during snack one day, an older female camper commented, "I didn't know what had gone in the other salsas, so I wanted to eat ours." In contrast to foods that campers helped prepare, they were often uncertain about or unfamiliar with the spices and seasonings in other dishes. Consequently, campers opted for the vegetable choice they were most confident they would enjoy, which in most cases was their selfprepared vegetable dish(es).

Hard work. The time and effort campers put into preparing vegetables at camp facilitated a sense of accomplishment and pride as they reaped the benefit of their efforts. One older female camper described how she correlated the effort she dedicated to tending the food with its improved taste:

I think because you worked hard it's better. It's kind of like earning money: A thief only feels guilty after he's stolen a certain amount, but an honest man who works that way feels like he's earned it. The money seems more valuable -[makes you feel] more rich. ... the food seems more different, special, exotic, valuable, costly because you've put your time and sweat into it.

Another younger female camper associated the idea of hard work with the theme of certainty:

There's a lot of effort going into it and everybody knows that, but whenever you buy stuff from the store, you don't know who's [grown and harvested] it, so you can't tell if a lot of effort's going into it or it's just some lazy bum working at the controls of some machine.

Campers believed the food they prepared was valuable because they put time and effort into preparing it. In turn, they associated this value with their improved preferences or choice for vegetables they helped prepare.

Pride and ownership. Relating to the theme of hard work and the corresponding value, campers expressed 
how they felt pride and ownership for the food they helped prepare. A younger female camper referenced the food she prepared: "It feels really good to know that you're the one who helped make it." These feelings were shared among campers of the older age group as well:

You just feel a sense of accomplishment. So even though it sometimes doesn't taste very good, it still tastes amazing to the person who makes it.

This camper noted that not everyone might have liked the food she helped prepare, but the pride and responsibility she generated in creating and eating her vegetables correlated with her liking of those particular vegetables. In turn, campers felt a responsibility toward vegetables they helped prepare, which may also have contributed to camper preferences and decisions during lunch- and snack-time activities.

Curiosity. Pertaining primarily to the theme of vegetable choice, but influencing preference, was the theme of curiosity. One camper noted:

I was interested in how it turned out because, typically, at home, we don't do salsa as much as we do guacamole ... it's just interesting to see what the difference was.

Other campers simply wanted to taste the product of their work, commenting, "I put a lot of work into it and I wanted to see how that tasted. If it was good," and, "I wanted to see if ... I liked it. I just like to try my own things." As indicated by these data, camper curiosity influenced their decision to eat food they prepared.

Experimentation. Many of the themes uncovered about campers' discussions of food were shared across the younger and older age groups. However, the idea of preparing food as a form of experimentation was limited to the older age group, which may provide insight into why campers often chose, but may not have preferred, vegetables they helped to prepare. One older girl explained how she enjoyed preparing vegetables in different ways but that those

experiences did not always turn out how she expected:

\section{I think we added too much of this and, even though it looks pretty, it's not exactly wonderful tasting. Maybe we should have boiled the green beans, not sautéed them.}

Another older camper noted how the ability to test new combinations was not something she could do at home: "I try a lot of new recipes because ... I don't really get to experiment with food a lot."

The older campers, more so than the younger campers, saw the opportunity to prepare food as a time to investigate new flavors. They were unsure whether or not they would like those flavors. Because of this approach, older campers may have had less certainty with regard to the flavors of the foods they prepared, as well as a greater likelihood that the food they prepared would not suit their taste. Although this postulate cannot be confirmed or rejected, a possible explanation for why the older campers chose their vegetables more frequently-but did not quantitatively prefer these vegetables more - may have been because the older campers used lunchtime as an opportunity to experiment with their food to a greater extent than the younger individuals; this, at times, resulting in undesirable flavors.

\section{DISCUSSION}

There is a current need in the US for methods to sustainably increase vegetable intake among children. The primary objective of this study was to examine how involvement in the vegetable growing, harvesting, and preparation processes might influence preference and choice, particularly among youth aged 8-14 years. Evident in aspects of both the qualitative and quantitative datasets, the younger campers preferred and chose more of the vegetables they helped prepare, except for carrots. Campers noted they enjoyed camp vegetables more than those purchased from a store, as they were fresher. The campers also expressed an added interest in eating vegetables for which they had found innovative new ways to prepare. Although vegetable preference increased across the camp session for zucchini, kale, and tomatoes, there was no significant preference increase for carrots and green beans. The lack of increase in preference for carrots and greens beans may be at least partially explained by high baseline preference scores-there was little or no room for improvement from baseline. This study's qualitative findings illustrate themes of agency, pride, and ownership are pertinent both in garden-based activities and in food preparation such as cooking and cutting. In addition, this research presents themes such as curiosity toward trying food children prepare by themselves; such perspectives rarely appear in other research.

Given that research was conducted in situ at a children's camp, the research participants could not be removed from the day-to-day planned activities to serve as a control group. This allowed for maintenance of face validity, as completion of the research did not intrude on the camp activities. The lack of a control group is accepted in similar in situ research situations in which the addition of a control group would exclude certain participants from available or desirable activities. $^{35-37,42}$

The improvement in preference as a result of garden-based interventions aligns with previous research on the matter. ${ }^{20,21}$ Earlier research on children's vegetable consumption and related behaviors often involved quantitative measures of preference, choice, and consumption. ${ }^{43,44}$ Nonetheless, several studies have qualitatively identified that children feel a sense of agency and pride over their food when working in a garden, ${ }^{20,45}$ and control over food-related decisions have been positively associated with vegetable consumption. ${ }^{46,47}$

Quantitatively, these studies present results aligned with the emerging field of research on how personal involvement impacts outcomes. This phenomenon, now referred to as the IKEA effect (named after the build it yourself furniture store IKEA), addresses how the value individuals give a product increases when they are involved in the building or 
preparation. ${ }^{48}$ Research into the IKEA effect originally addressed the production of nonconsumable physical objects, such as furniture, origami, or Lego sets, ${ }^{48,49}$ but is now beginning to move into the realm of food. ${ }^{50}$ Van der Horst et $\mathrm{al}^{51}$ noted that children who helped their parents prepare a meal of salad, chicken, pasta, and cauliflower consumed significantly more salad and chicken but not pasta or cauliflower than kids who were not involved. Similarly, increased frequency in food preparation at home has been correlated with increased vegetable and fruit consumption. ${ }^{52}$ The present findings are consistent with studies addressing the value of cooking ${ }^{28}$ or involvement via gardening ${ }^{53}$ on vegetable consumption. Although the data from this study are supported by this past research on the benefits of involvement, the data presented here provide several novel findings on various elements of involvement in vegetable preparation, including harvesting, cooking, cutting, and seasoning. First, previous research has focused on consumption of a variety of food types, such as vegetables, fruits, carbohydrates, healthy food in general, and so on, whereas this research focused on the impact of involvement on vegetable preference alone, which is a notable finding given low fruit and vegetable consumption among US adolescents. Furthermore, this investigation analyzed how specific elements of involvement, both in the garden (harvesting) and in the kitchen setting (cutting, cooking, and seasoning), influence vegetable preferences. This study provides evidence suggesting that increasing vegetable preference among children aged 8 -10 years is feasible through involving these adolescents in gardening and cooking-related activities.

In contrast to the younger age group, the changes in preferences for vegetables they helped to prepare did not reach statistical significance in the older age group. Although this study cannot conclude the reason for the said difference, there may have been internal, age-based differences between the older and younger camp group that manifested in differences in preferences. For example, neophobia (fear of trying novel foods) decreases with age, suggesting that the older campers may have been more likely to try their own unique, but not necessarily tastier, foods. ${ }^{54}$ In addition, because of the smaller sample size for the older age group, the study may not have had the power to detect differences.

This study had limitations in terms of accurately determining the strengths of the associations found. It was not ethical to randomize children to a situation in which one group was involved in preparing food, whereas the other was not, preventing the formation of a control group. Furthermore, the children had varying prior exposure to certain vegetables and experience with food preparation. Finally, this study measured vegetable preference rather than vegetable consumption. However, because preference is correlated with consumption, the measurement preference was seen as a reasonable proxy for consumption and a way to address the impact of involvement in preparation. ${ }^{55,56}$

\section{IMPLICATIONS FOR RESEARCH AND PRACTICE}

Direct, firsthand involvement with food preparation, in particular in a garden-based setting, may provide an accessible approach to improving children's vegetable preferences, especially among children aged 8 -10 years. In a home setting, involving children in preparing healthy food is recommended. Such approaches may be particularly advantageous for initiating uptake of healthier foods within a younger age cohort, with less impactful outcomes among older children. Furthermore, as gardening programs become increasingly popular in the US, these data support the need for further examination regarding how adding a cooking component to existing gardening programs impacts vegetable preference and consumption.

\section{ACKNOWLEDGMENTS}

This study was supported through Stanford University's Department of Human Biology and a Major Grant through the Office for the Vice Provost for Undergraduate Education at Stanford University. Data were originally from the thesis of the primary author.

\section{SUPPLEMENTARY DATA}

Supplementary data related to this article can be found at https://doi. org/10.1016/j.jneb.2021.08.009.

\section{REFERENCES}

1. Nadeau KJ, Maahs DM, Daniels SR, Eckel RH. Childhood obesity and cardiovascular disease: links and prevention strategies. Nat Rev Cardiol. 2011; 8:513-525.

2. Ogden CL, Carroll MD, Kit BK, Flegal KM. Prevalence of obesity and trends in body mass index among US children and adolescents. JAMA. 2012;307:483490.

3. Pinhas-Hamiel O, Zeitler P. The global spread of type 2 diabetes mellitus in children and adolescents. I Pediatr. 2005; 146:693-700.

4. Weiss R, Caprio S. The metabolic consequences of childhood obesity. Best Pract Res Clin Endocrinol Metab. 2005;19:405-419.

5. Boumtje PI, Huang CL, Lee JY, Lin BH. Dietary habits, demographics, and the development of overweight and obesity among children in the United States. Food Policy. 2005;30:115-128.

6. Vos MB, Kaar JL, Welsh JA, et al. Added sugars and cardiovascular disease risk in children: A scientific statement from the American Heart Association. Circulation. 2017;135:e1017-e1034.

7. Bazzano LA, He J, Ogden LG, et al. Fruit and vegetable intake and risk of cardiovascular disease in US adults: the first National Health and Nutrition Examination Survey Epidemiologic Follow-up Study. Am J Clin Nutr. 2002; 76:93-99.

8. World Cancer Research Fund. Diet, Nutrition, Physical Activity and Cancer: a Global Perspective. World Cancer Research Fund; 2018. https://www.wcrf.org/dietandcancer. Accessed March 3, 2021.

9. Epstein LH, Gordy CC, Raynor HA, Beddome M, Kilanowski CK, Paluch $\mathrm{R}$. Increasing fruit and vegetable intake and decreasing fat and sugar intake in families at risk for childhood obesity. Obes Res. 2001;9:171-178.

10. Field AE, Gillman MW, Rosner B, Rockett HR, Colditz GA. Association 
between fruit and vegetable intake and change in body mass index among a large sample of children and adolescents in the United States. Int J Obes Relat Metab Disord. 2003;27:821-826.

11. He K, Hu FB, Colditz GA, Manson JE, Willett WC, Liu S. Changes in intake of fruits and vegetables in relation to risk of obesity and weight gain among middle-aged women. Int $J$ Obes Relat Metab Disord. 2004;28:1569-1574.

12. McCrory MA, Fuss PJ, McCallum JE, et al. Dietary variety within food groups: association with energy intake and body fatness in men and women. Am J Clin Nutr. 1999;69:440-447.

13. Miedema MD, Petrone A, Shikany JM, et al. Association of fruit and vegetable consumption during early adulthood with the prevalence of coronary artery calcium after 20 years of follow-up: the Coronary Artery Risk Development in Young Adults (CARDIA) Study. Circulation. 2015;132:1990-1998.

14. Slavin JL, Lloyd B. Health benefits of fruits and vegetables. $A d v$ Nutr. 2012;3:506-516.

15. Mikkilä V, Räsänen L, Raitakari OT, Pietinen P, Viikari J. Consistent dietary patterns identified from childhood to adulthood: the cardiovascular risk in Young Finns Study. $\mathrm{Br} J$ Nutr. 2005;93:923-931.

16. US Department of Agriculture and US Department of Health and Human Services. Dietary Guidelines for Americans, 2020-2025. 9th ed. US Department of Agriculture and US Department of Health and Human Services; 2020.

17. US Department of Agriculture, Agricultural Research Service. What We Eat in America, NHANES 2015-2016 data tables. https://www.ars.usda.gov/ ARSUserFiles/80400530/pdf/fped/ Table_1_FPED_GEN_1516.pdf. Accessed May 14, 2021.

18. Lorson BA, Melgar-Quinonez HR, Taylor CA. Correlates of fruit and vegetable intakes in US children. J Am Diet Assoc. 2009;109:474-478.

19. Garcia AL, Reardon R, McDonald M, Vargas-Garcia EJ. Community interventions to improve cooking skills and their effects on confidence and eating behaviour. Curr Nutr Rep. 2016;5: 315-322.

20. Heim S, Stang J, Ireland M. A garden pilot project enhances fruit and vegetable consumption among children. J Am Diet Assoc. 2009;109:1220-1226.
21. Ratcliffe MM, Merrigan KA, Rogers BL, Goldberg JP. The effects of school garden experiences on middle schoolaged students' knowledge, attitudes, and behaviors associated with vegetable consumption. Health Promot Pract. 2011;12:36-43.

22. Morgan PJ, Warren JM, Lubans DR, Saunders KL, Quick GI, Collins CE. The impact of nutrition education with and without a school garden on knowledge, vegetable intake and preferences and quality of school life among primary-school students. Public Health Nutr. 2010;13:1931-1940.

23. Morris J, Neustadter A. ZidenbergCherr S. First-grade gardeners more likely to taste vegetables. Calif Agric. 2001;55:43-46.

24. Parmer SM, Salisbury-Glennon J, Shannon D, Struempler B. School gardens: an experiential learning approach for a nutrition education program to increase fruit and vegetable knowledge, preference, and consumption among second-grade students. J Nutr Educ Behav. 2009;41:212-217.

25. McAleese JD, Rankin LL. Gardenbased nutrition education affects fruit and vegetable consumption in sixth grade adolescents. J Am Diet Assoc. 2007;107:662-665.

26. van der Horst $\mathrm{K}$, Mathias KC, Prieto Patron A, Allirot X. Art on a plate: a pilot evaluation of an international initiative designed to promote consumption of fruits and vegetables by children. J Nutr Educ Behav. 2019;51:919-925.

27. Jarpe-Ratner E, Folkens S, Sharma S, Daro D, Edens NK. An experiential cooking and nutrition education program increases cooking self-efficacy and vegetable consumption in children in grades 3-8. J Nutr Educ Behav. 2016;48:697-705.e1.

28. Cunningham-Sabo L, Lohse B. Cooking with kids positively affects fourth graders' vegetable preferences and attitudes and self-efficacy for food and cooking. Child Obes. 2013; 9:549-556.

29. Lautenschlager L, Smith C. Beliefs, knowledge, and values held by inner-city youth about gardening, nutrition, and cooking. Agric Hum Values. 2007;24:245.

30. Reicks M, Trofholz AC, Stang JS, Laska MN. Impact of cooking and home food preparation interventions among adults: outcomes and implications for future programs. J Nutr Educ Behav. 2014; 46:259-276.
31. Hutchinson J, Watt JF, Strachan EK, Cade JE. Evaluation of the effectiveness of the Ministry of Food cooking programme on self-reported food consumption and confidence with cooking. Public Health Nutr. 2016; 19:3417-3427.

32. Irwin LG, Johnson J. Interviewing young children: explicating our practices and dilemmas. Qual Health Res. 2005;15:821-831.

33. Ando H, Cousins R, Young C. Achieving saturation in thematic analysis: development and refinement of a codebook. Comprehensive Psychology. 2014;3:1-7.

34. Faux SA, Walsh M, Deatrick JA. Intensive interviewing with children and adolescents. West J Nurs Res. 1988; 10:180-194.

35. Bean MK, Brady Spalding B, Theriault E, Dransfield KB, Sova A, Dunne Stewart M. Salad bars increased selection and decreased consumption of fruits and vegetables 1 month after installation in title I elementary schools: a plate waste study. J Nutr Educ Behav. 2018;50:589-597.

36. Garcia AL, Vargas E, Lam PS, Shennan DB, Smith F, Parrett A. Evaluation of a cooking skills programme in parents of young children-a longitudinal study. Public Health Nutrition. 2014;17:1013-1021.

37. Overcash F, Ritter A, Mann T, et al. Impacts of a vegetable cooking skills program among low-income parents and children. J Nutr Educ Behav. 2018;50:795-802.

38. Saldana J. The Coding Manual for Qualitative Researchers. Sage Publications; 2015.

39. Mattys L, Noens I, Evers K, Baeyens D. "Hold me tight so I can go it alone": developmental themes for young adults with autism spectrum disorder. Qual Health Res. 2018;28:321-333.

40. Miles MB, Huberman AM, Saldaña J. Qualitative Data Analysis. Sage Publications; 2013.

41. Taylor SJ, Bogdan R, DeVault M. Introduction to Qualitative Research Methods: A Guidebook and Resource. John Wiley \& Sons; 2015.

42. Kaiser LL, Sadeghi B, Tseregounis IE, et al. Attitudes and social norms are related to attendance at childhood obesity prevention classes in a rural Mexican-heritage community. J Nutr Educ Behav. 2018;50:824-828.

43. Duncan MJ, Eyre E, Bryant E, et al. The impact of a school-based gardening intervention on intentions and 
behaviour related to fruit and vegetable consumption in children. $J$ Health Psychol. 2015;20:765-773.

44. Jaenke RL, Collins CE, Morgan PJ, Lubans DR, Saunders KL, Warren JM. The impact of a school garden and cooking program on boys' and firls' fruit and vegetable preferences, taste rating, and intake. Health Educ Behav. 2012;39:131-141.

45. Libman K. Growing youth growing food: how vegetable gardening influences young people's food consciousness and eating habits. Appl Environ Educ Commun. 2007;6:87-95.

46. Wardle J, Carnell S, Cooke L. Parental control over feeding and children's fruit and vegetable intake: how are they related? J Am Diet Assoc. 2005;105:227-232.

47. Orlet Fisher J, Mitchell DC, SmiciklasWright H, Birch LL. Parental influences on young girls' fruit and vegetable, micronutrient, and fat intakes. $J \mathrm{Am}$ Diet Assoc. 2002;102:58-64.
48. Norton MI, Mochon D, Ariely D. The IKEA effect: when labor leads to love. J Consum Psychol. 2012;22:453-460.

49. Marsh L, Kanngiesser P, Hood B. When and how does labour lead to love? The ontogeny and mechanisms of the IKEA effect. Cognition. 2018;170:245-253.

50. Dohle S, Rall S, Siegrist M. I cooked it myself: preparing food increases liking and consumption. Food Quality and Preference. 2014;33:14-16.

51. van der Horst K, Ferrage A, Rytz A. Involving children in meal preparation. Effects on food intake. Appetite. 2014;79:18-24.

52. Chu YL, Farmer A, Fung C, Kuhle S, Storey KE, Veugelers PJ. Involvement in home meal preparation is associated with food preference and self-efficacy among Canadian children. Public Health Nutr. 2013;16:108-112.

53. Lineberger SE, Zajicek JM. School gardens: can a hands-on teaching tool affect students' attitudes and behaviors regarding fruit and vegetables? Horttechnology. 2000;10:593-597.

54. Koivisto UK, Sjödén PO. Food and general neophobia in Swedish families: arent-child comparisons and relationships with serving specific foods. Appetite. 1996;26:107-118.

55. Baxter SD, Thompson WO. Fourthgrade children's consumption of fruit and vegetable items available as part of school lunches is closely related to preferences. $J$ Nutr Educ Behav. 2002;34:166-171.

56. Gibson EL, Wardle J, Watts CJ. Fruit and vegetable consumption, nutritional knowledge and beliefs in mothers and children. Appetite. 1998;31:205-228.

\section{ORCID}

Isabelle L. Crary: http://orcid.org/ 0000-0003-0820-3446 